Joaquim Eurico Valentim Oliveira José Fernando Vasconcelos Cabral Pinto ${ }^{2}$ Rita Manuela de Almeida Barros ${ }^{2}$

${ }^{1}$ Unidade de Investigação em Educação e Tecnologias Educativas, Instituto Piaget. Vila Nova de Gaia, Portugal

${ }^{2}$ Research in Education and Community Intervention. Instituto Piaget. Vila Nova de Gaia, Portugal.

Contato:

Rita Manuela de Almeida Barros

E-mail:

rita.barros@gaia.ipiaget.pt

* Optou-se por manter a grafia original de Portugal apresentada pelos autores.

O trabalho é baseado em tese de doutoramento de Joaquim Eurico Valentim Oliveira, intitulada A formação profissional no mercado transicional em Portugal, defendida em 2012 na Universidade de Santiago de Compostela, Galicia, Espanha.

$\mathrm{O}$ estudo foi apresentado no $\mathrm{XX}^{\mathrm{O}}$ Colóquio da Association Francophone Internationale de Recherche Scientifique en Education (Afirse), realizado em Lisboa, em 2013.

Os autores declaram não haver conflitos de interesse e que o trabalho não foi subvencionado.

Recebido: 27/09/2012

Revisado: 31/07/2013

Aprovado: 26/09/2013

\section{A formação profissional e a perceção de saúde mental dos trabalhadores: estudo comparativo em situação de (des)emprego*}

\author{
Vocational training and workers' mental health awareness: a \\ comparative study in an (un)employment situation
}

\section{Resumo}

Introdução: a atual reconfiguração do mercado de trabalho surge no contexto da emergência de novas realidades económicas, históricas e sociais, pautadas pela complexidade, incerteza, aceleração e imprevisibilidade. Objetivo: face às exigências de adaptação psicossocial implícitas nesse contexto, o presente estudo pretendeu diferenciar a auto perceção dos trabalhadores no que concerne à sua saúde mental, em função da frequência em programas de formação profissional, quer se trate de trabalhadores em situação de emprego ou de desemprego. Métodos: Para o efeito, foram inquiridos 210 sujeitos. Na avaliação da autoperceção de saúde mental foi utilizada a adaptação portuguesa do General Health Questionnaire (G.H.Q.- 28). Resultados: os resultados evidenciaram diferenças significativas no que concerne à autoperceção de saúde mental dos trabalhadores em função da sua frequência em programas de formação profissional, mostrando melhor situação entre os que frequentam cursos, sejam homens ou mulheres $(t=-4,503 ; p<0,001$ e $t=-4,737 ; p<0,001)$, empregados ou desempregados $(t=-5,551 ; p<0,001$ e $t=$ $-2,458 ; p<0,05)$. Verificou-se ainda que os trabalhadores empregados que continuaram a aderir a ofertas de formação profissional, apresentam uma auto perceção de saúde mental significativamente mais favorável $(\mathrm{t}=-5,138 ; \mathrm{p}<0,001)$. Conclusão: os resultados permitem supor que o envolvimento dos trabalhadores em acções de formação profissional funciona como elemento protetor da sua saúde mental.

Palavras-Chave: mercado trabalho; formação profissional; saúde mental.

\begin{abstract}
Background: the present reconfiguration of the labor market arises in the context of new economic, social and historical events, characterized by complexity, uncertainty, unpredictability and acceleration. Objective: given the psychosocial adaptation required by this context, the present study intends to distinguish workers' self-perception concerning their mental health, according to their participation in vocational training programs, whether they are employed or unemployed. Methods: to achieve this purpose we interviewed 210 workers. To evaluate self-perceived mental health, a Portuguese adaptation of the General Health Questionnaire (G.H.Q.-28) was used . Results: results evidenced significant differences in workers' mental health self-perception. These differences were related to the workers' attendance in vocational programs, with advantage for those attending courses, whether men or women $(t=-4.503 ; p<0.001$ and $t=-4.737 ; p<0.001)$, employed or unemployed ( $t=-5.551 ; p<0.001$ and $t=-2.458 ; p<0.05)$. It was also found that the employed workers who continued to join vocational training offers had a significantly more favorable mental health self perception ( $t=-5.138 ; p<0.001)$. Conclusion: results support the hypothesis that involvement in vocational training activities functions as a protective element for workers' mental health.
\end{abstract}

Keywords: labor market; vocational training; mental health. 


\section{Introdução}

\section{A pertinência e atualidade do tema}

Na sequência da evolução do capitalismo, o mercado de trabalho tem vindo a sofrer alterações estruturais, cujos efeitos são visíveis sobretudo pela elevada oferta de postos de trabalho precários, isto é, empregos temporários, mal remunerados e geralmente sem direitos de proteção social (GAZIER, 1999; STIGLITZ, 2004). Álvaro, Torregrosa e Luque (1992, p.23), numa investigação referente à saúde mental da população ativa espanhola, sustentada numa amostra de 1253 sujeitos, constataram que o grau de deterioração psicológica observado nos desempregados era semelhante ao dos trabalhadores temporários, concluindo que "a estabilidade no emprego é uma circunstância adicional no conjunto de fatores que incidem no bem-estar psicológico".

Se é verdade que o stress laboral não é um assunto inédito (HARNOIS; GABRIEL, 2000), também é verdade que aquele que é experienciado na atualidade, por força do aumento significativo da volatilidade das instituições laborais, não tem termo de comparação com o do passado. É na constatação desta evolução negativa que assenta a pretensão de pertinência desta investigação. Com ela procura-se dar um passo em frente no que concerne à identificação de condições que possam minorar os efeitos nefastos dos níveis crescentes de stress laboral na atualidade. Assim, decidiu-se colocar a questão de saber se a frequência em cursos de formação profissional concorre ou não para tal desiderato.

\section{A saúde mental dos trabalhadores no contexto laboral} de incerteza e o papel da formação profissional.

De acordo com a Organização Mundial de Saúde (2001): "As políticas devem maximizar as oportunidades de emprego para toda a população e reter as pessoas na força de trabalho, particularmente por causa da associação entre perda de emprego e aumento do risco de transtorno mental e suicídio".

A centralidade do emprego foi sempre uma questão de importância indiscutível relativamente ao cumprimento das funções psicossociais de bem-estar (BLANCH, 1990). Sucede que os empregos de carreira, que eram emblemáticos da sociedade fordista, foram sendo substituídos por empregos transitórios, de curta duração, nas mais diferentes áreas profissionais, os quais, por serem segmentados no tempo, já não cumprem cabalmente todas aquelas funções. Resultam daí hiatos de sociabilidade profissional que devem ser colmatados, ainda que de forma artificial, com vista à salvaguarda do bem-estar psicológico.
Ressalta, pois, a partir da década de 90, o interesse renovado pela formação ao longo da vida (FERNANDÉZ, 2005), cujo valor para o contexto presente foi assinalado no âmbito da União Europeia (1995), designadamente pelo Parlamento Europeu e o Conselho, que realçaram a sua importância em aspetos como a empregabilidade, isto é, a adaptação permanente dos ativos a um mercado de trabalho em transição (Decisão n ${ }^{\circ}$ 2493/95/CE do Parlamento Europeu e do Conselho, de 23 de Outubro de 1995). O caráter adaptável dos cursos de formação profissional pós-fordista às demandas variáveis do mercado de trabalho e a consequente potenciação da empregabilidade de quem os frequenta deixam antever a existência de benefícios psicossociais ainda por avaliar, nomeadamente na prevenção dos efeitos corrosivos da insegurança e da incerteza sobre a saúde mental dos trabalhadores. Numa investigação relativa ao estudo do efeito das condições ocupacionais nas "vivências de sofrimento" no trabalho, Ferreira (2002, p. 181) realçou a importância da formação profissional para a moderação da ansiedade dos próprios trabalhadores em exercício, facilitando "o vínculo entre estes e a unidade organizacional, [...], o desenvolvimento de sentimentos de pertença, [...] e, sobretudo, de sentir-se bem na organização". Isto quer dizer que a frequência da formação profissional pode funcionar como uma eventual estratégia de $\mathrm{CO}^{-}$ ping para os ativos (MARTINS, 2004), entendendo o coping como o conjunto de esforcos comportamentais e cognitivos, em mudanca constante, que visam gerir exigências internas e externas específicas, consideradas como excedendo os recursos pessoais (RIBEIRO, 2005). Desta forma, a formação profissional poderá ser uma via alternativa para enfrentar a situação de stress vivida diariamente no mundo do trabalho, podendo também servir de complemento ao emprego no que concerne à tarefa de assegurar o cumprimento de funções psicossociais de bem-estar que o emprego já não garante na íntegra.

\section{A relevância da sanidade mental num contexto de stress laboral: dimensão do problema.}

A relação entre a debilidade das condições laborais oferecidas pelo mercado de emprego e a vulnerabilidade à doença mental da população ativa tem sido objeto de reflexão por parte de entidades internacionais responsáveis pela saúde e desenvolvimento económico. A discussão centra-se sobretudo no interesse de mitigar os problemas mentais inerentes ao stress laboral, na medida em que os mesmos estão associados a uma terapêutica com um custo demasiado expressivo no erário público dos países. Porém, o stress laboral e os seus efeitos na saúde mental também se repercutem na criação de riqueza, na medida em que contribuem para o aumento signi- 
ficativo do número de baixas médicas, e, consequentemente, para a queda da produtividade do trabalho.

Atendendo à atualidade que a conjuntura económica restituiu ao tema, alguns autores despertaram novamente para a gravidade das consequências psicológicas dos problemas de stress laboral, o que os levou a desenvolver estudos orientados para a divulgação científica, junto das autoridades públicas, dos seus efeitos maléficos. Conforme os resultados da investigação de Glina et al. (2001), cujo objetivo foi averiguar a existência de um nexo de causalidade entre as condições de trabalho e os danos na saúde mental, estão associadas a condições de trabalho adversas e a dificuldades na organização e gestão laboral manifestações de medo, ansiedade, depressão, nervosismo, tensão, fadiga, mal-estar, perda de apetite, distúrbios de sono, distúrbios psicossomáticos.

Se uma boa saúde mental estimula a capacidade de trabalho e a produtividade, em contraposição, a precariedade, o espectro do desemprego e a mobilidade forçada geram desequilíbrios psíquicos, absentismo por doença, aumento de custos e diminuição do desenvolvimento económico. São também conhecidas as relações entre desemprego, perda de autoestima, humor depressivo, deterioração psicológica geral e ansiedade (ÁLVARO, 1992; BANKS, 1992; RIPOLL; RODRIGUEZ; PEIRÓ, 1996; BUENDIA, 2001; ÁLVARO; GARRIDO, 2002), de tal forma que as iniciativas de reintegração dos desempregados no mercado de trabalho são desejáveis, não apenas por razões de ordem económica, mas como estratégia de promoção da coesão social.

Tendo por pano de fundo esta realidade, o estudo empírico que apresentamos a seguir tentou auscultar se a frequência em cursos de formação profissional distingue os níveis de saúde mental dos indivíduos. Ou seja, pretendemos verificar se os participantes em formação profissional têm vantagens de saúde mental em relação aos demais. Por pragmatismo metodológico, que nos levou a isolar uma só variável independente, partimos do pressuposto de que os trabalhadores (empregados e desempregados) que cumprem a condição de terem frequentado um curso de formação profissional há menos de um ano apresentam uma perceção mais favorável em relação à sua saúde mental, quando comparados com os sujeitos que não se encontram nessa condição. Dito de outra forma, pretendeu-se averiguar se os trabalhadores (empregados e desempregados) que realizaram formação profissional revelam alguma vantagem em termos de benefício para a saúde mental, comparativamente aos demais. No fundo, o interesse reside em saber se a formação profissional constitui (ou não) uma estratégia de coping, isto é, uma "ferramenta" que, ao permitir enfrentar o stress laboral, conduza à minimização dos danos de saúde mental dos trabalhadores, associados à incerteza quotidiana do mercado de trabalho.

\section{Metodologia}

Apresentamos um estudo transversal, comparativo, não experimental de natureza quantitativa.

\section{Amostra}

No presente estudo recorremos a uma amostragem não probabilística, definida por conveniência, tendo em conta a acessibilidade aos sujeitos. Tratando-se de uma amostragem por conveniência, os sujeitos que integraram o estudo foram seleccionados tendo em conta a sua disponibilidade e motivação para o mesmo. As preocupações com a proporcionalidade em relação às categorias dos grupos estabelecidos traduziram-se no equilíbrio da dimensão dos grupos, em termos idade, curso de formação (com ou sem curso) e situação profissional (empregado ou desempregado). Assim, 49,1\% da amostra integrava trabalhadores sem curso de formação profissional, $45,7 \%$ eram do sexo masculino e $52,9 \%$ encontravam-se na situação de desemprego. Foram inquiridos 210 trabalhadores residentes em contexto suburbano no norte de Portugal, uma das zonas do país mais afetadas pelo flagelo do desemprego. Todos os sujeitos residiam numa das freguesias do município de Vila Nova de Gaia, distrito do Porto, e encontravam-se em idade ativa para o trabalho. Dos 216 sujeitos inicialmente seleccionados, 6 acabaram por ser excluídos por sofrerem de doenças físicas crónicas. Foram também critérios de exclusão da amostra a opção pelo abandono do mundo laboral por preferência de actividades domésticas e as situações de toxicodependência, alcoolismo e luto. Estas medidas de precaução foram consideradas para evitar o enviesamento dos resultados finais. Os sujeitos que frequentaram curso de formação profissional foram inquiridos até 12 meses após a finalização do mesmo.

\section{Instrumento.}

Para além do questionário sociodemográfico que sustentou a caraterização da amostra, os sujeitos preencheram a adaptação portuguesa do General Health Questionnaire (G.H.Q.-28), isto é, uma escala de saúde mental. Na sua versão original, o General Health Questionnaire (G.H.Q.-28) é uma escala de avaliação de distúrbios psiquiátricos não psicóticos, composta por quatro subescalas que integram 28 itens distribuídos por quatro fatores (GOLDBERG; HILLIER, 1979). A resposta é dada numa escala de "0" a "3", 
sendo que a nota total do questionário varia entre “0” " 84 ". A valores mais elevados corresponde uma auto-percepção de saúde de mental mais negativa. A versão portuguesa da escala total G.H.Q.-28 apresenta uma boa consistência interna (alpha de Cronbach de 0,922), assim como as subescalas que a compõem: sintomas somáticos (alpha de Cronbach de 0,825), ansiedade/insónia (alpha de Cronbach de 0,873), disfunção social (alpha de Cronbach de 0,873) e depressão severa (alpha de Cronbach de 0,838).

Embora a sua designação remeta para um questionário de saúde geral, na verdade trata-se de um recurso avaliativo da saúde mental,

“ (...) concebido para identificar quer a incapacidade para realizar as actividades que são usuais numa pessoa saudável, quer o aparecimento de fenômenos stressantes novos. Portanto, não visa detetar traços estáveis, mas sim quebras no funcionamento usual. Além disso, não é um questionário de saúde geral, mas sim um questionário apropriado para avaliar a saúde mental ou o bem-estar psicológico" (RIBEIRO; ANTUNES, 2003, p. 37).

A opção por este instrumento sustentou-se em estudos prévios realizados em Portugal (MONTEIRO, 2011; RIBEIRO; ANTUNES, 2003) nos quais foram confirmadas as qualidades psicométricas da escala e avançada a possibilidade da sua utilização em populações adultas em contextos não clínicos portugueses.

\section{Procedimentos e análise estatística.}

A coleta de dados foi realizada até 12 meses após o terminus da conclusão do curso de formação profissional realizado. Aos participantes foi garantido o anonimato e a confidencialidade individual dos resultados, respeitando-se os aspectos éticos de acordo com a Declaração de Helsinque. A análise estatística dos dados da amostra foi realizada através do SPSS Statistical Package for the Social Sciences 19.0 - e os procedimentos estatísticos adotados passaram pela estatística descritiva e inferencial. Considerando a distribuição normal dos resultados e confirmada a homogeneidade de contingências dos grupos em comparação, através do Teste de Levene ( $p>0,05)$, optamos pela estatística paramétrica. As comparações entre grupos foram efetuadas por recurso ao t-test para amostras independentes. Admitimos um nível de significância estatística p<0,05 (MARÔCO, 2010; MARÔCO; BISPO, 2005).

A análise comparativa da saúde mental dos sujeitos estabeleceu-se em função da conclusão de curso de formação profissional há menos de um ano, da situação de (des)emprego e da variável sexo. Dito de outro modo, procedeu-se à comparação dos níveis médios de saúde mental entre os trabalhadores (empregados e desempregados), com e sem curso de formação profissional, tendo sido também levada em consideração a variável sexo.

\section{Resultados}

A caracterização das variáveis de sexo, idade, situação de trabalho e nível educacional nos dois grupos (com e sem curso de formação) é apresentada na Tabela 1 .

Tabela 1 Caracterização das variáveis de sexo, idade, situação de trabalho e nivel educacional entre os dois grupos (com e sem curso de formação). Junta de Freguesia de Canelas, Vila Nova de Gaia, Portugal. Fevereiro de 2008 a fevereiro de 2009

\begin{tabular}{|c|c|c|c|}
\hline & $\begin{array}{c}\text { Sujeitos com Formação } \\
\text { Profissional }(\mathrm{N}=107)\end{array}$ & $\begin{array}{l}\text { Sujeitos sem Formação } \\
\text { Profissional }(N=103)\end{array}$ & Sig. \\
\hline Sexo & & & $c 2=1,492$ \\
\hline Homem & 44 & 52 & $(g \mid=1)$ \\
\hline Mulher & 63 & 51 & $\mathrm{p}=0,222$ \\
\hline Idade & $M=36,33 / D P=11,58$ & $M=36,26 / D P=11,63$ & \multirow{4}{*}{$\begin{array}{c}c 2=0,24 \\
(g \mid=2) \\
p=0,988\end{array}$} \\
\hline 16-29 anos & 40 & 39 & \\
\hline $30-50$ anos & 39 & 38 & \\
\hline+50 anos & 28 & 26 & \\
\hline Situação de Trabalho & & & \multirow{3}{*}{$\begin{array}{c}c 2=6,984 \\
(g \mid=1) \\
p=0,008\end{array}$} \\
\hline Empregado & 60 & 39 & \\
\hline Desempregado & 47 & 64 & \\
\hline \multicolumn{3}{|l|}{ Nível Educacional } & \multirow{4}{*}{$\begin{array}{c}c 2=2,585 \\
(g \mid=2) \\
p=0,275\end{array}$} \\
\hline Menos que escolaridade obrigatória & 14 & 21 & \\
\hline Escolaridade obrigatória & 48 & 47 & \\
\hline Mais do que a escolaridade obrigatória & 45 & 35 & \\
\hline
\end{tabular}


Relativamente à saúde mental dos trabalhadores da amostra global, com e sem curso de formação profissional, verifica-se que aqueles que concluíram curso de formação profissional revelaram uma auto perceção média de saúde mental (média $=29,24$; desvio padrão $=6,23)$, que é significativamente mais favorável $(\underline{t}=-6,626 ; p<0,001)$ do que a auto perceção de saúde mental dos trabalhadores que não frequentaram curso de formação profissional, cuja média foi de 35,35, com um desvio padrão de 7,1.

$\mathrm{O}$ valor do t-test para amostras independentes revelou que os trabalhadores do sexo masculino, com curso de formação profissional, apresentam uma perceção mais favorável em relação à sua saúde mental (média $=28,64$; desvio padrão $=7,58$ ), do que os trabalhadores do sexo masculino que não tiveram acesso à formação profissional (média $=35,5$; desvio padrão $=7,32), \underline{\mathrm{t}}=-4,503, \mathrm{p}<0,001$.

Se considerarmos o sexo feminino, os valores estatísticos obtidos apontam no mesmo sentido, isto é, verifica-se que as mulheres que realizaram curso de formação profissional apresentam uma auto perceção de saúde mental mais positiva (média $=29,67$; desvio padrão $=5,1$ ), do que as mulheres sem curso de formação profissional (média $=35,2$; desvio padrão=6,96), $\underline{\mathrm{t}}=-4,89737 ; \mathrm{p}<0,001$.

Com relação aos sujeitos que se encontram em situação de emprego, os resultados indicam diferenças estatisticamente significativas entre a auto perceção de saúde mental dos trabalhadores que realizaram curso de formação profissional (média $=26,52$; desvio padrão $=5,1$ ) e a auto perceção de saúde mental dos trabalhadores que não realizaram curso de formação profissional (média = 32,33; desvio padrão $=4,88$ ), com vantagem para os primeiros, $\underline{\mathrm{t}}=-5,551 ; \mathrm{p}<0,001$.

Ao comparar os trabalhadores desempregados com curso de formação profissional com os trabalhadores desempregados sem curso de formação profissional, verifica-se que existem diferenças estatisticamente significativas entre os dois grupos $(\mathrm{t}=$ 2,$458 ; \mathrm{p}<0,05$ ), que assinalam uma auto perceção de saúde mental mais favorável dos primeiros (média $=33,45$; desvio padrão $=6,6$ ) face aos segundos (média $=36,76$; desvio padrão=7,37).

Por último, verificamos que os trabalhadores empregados que, após a situação de emprego, continuaram a aderir a ofertas no âmbito da formação profissional, apresentam uma auto perceção de saúde mental significativamente mais favorável (média= 26,67; desvio padrão=6,35), do que aqueles que não aderiram (média $=31,71$; desvio padrão $=3,29$ ), $\underline{\mathrm{t}}=$ $-5,138 ; \mathrm{p}<0,001$.

A Tabela 2 apresenta os valores descritivos e comparativos da auto-perceção de saúde mental dos trabalhadores com e sem curso de formação profissional, em função do sexo, situação laboral e continuidade da formação para os trabalhadores empregados.

Tabela 2 Valores descritivos e comparativos da auto-perceção de saúde mental em homens e mulheres com e sem formação profissional, trabalhadores empregados e desempregados com e sem formação profissional e trabalhadores empregados com (des)continuidade em termos de formação profisssional. Junta de Freguesia de Canelas, Vila Nova de Gaia, Portugal. Fevereiro de 2008 a fevereiro de 2009

\begin{tabular}{lccccc}
\hline & $N$ & Média & Desvio padrão & t-test & $p$ \\
\hline Homens sem formação profissional & 52 & 35,50 & 7,320 & & \\
Homens com formação profissional & 44 & 28,64 & 7,582 & $-4,503$ & 0,000 \\
Mulheres sem formação profissional & 51 & 35,20 & 6,957 & & \\
Mulheres com formação profissional & 63 & 29,67 & 5,105 & $-4,737$ & 0,000 \\
$\begin{array}{l}\text { Trabalhadores empregados com curso de } \\
\text { formação profissional }\end{array}$ & 60 & 26,52 & 5,229 & & 0,000 \\
$\begin{array}{l}\text { Trabalhadores empregados sem curso de } \\
\text { formação profissional }\end{array}$ & 39 & 32,33 & 4,879 & & \\
$\begin{array}{l}\text { Trabalhadores desempregados com curso de } \\
\text { formação profissional }\end{array}$ & 49 & 33,45 & 6,605 & & \\
$\begin{array}{l}\text { Trabalhadores desempregados sem curso de } \\
\text { formação profissional }\end{array}$ & 62 & 36,76 & 7,370 & $-2,458$ & 0,016 \\
$\begin{array}{l}\text { Trabalhadores empregados a frequentar } \\
\text { formação profissional }\end{array}$ & 57 & 26,67 & 6,348 & & \\
$\begin{array}{l}\text { Trabalhadores empregados a não frequentar } \\
\text { formação profissional }\end{array}$ & 42 & 31,71 & 3,293 & $-5,138$ & 0,000 \\
\hline
\end{tabular}




\section{Discussão e Considerações finais}

Verificamos que a auto perceção de saúde mental, por parte dos trabalhadores, é mais favorável após a frequência e conclusão de curso de formação profissional, quer na situação em que os sujeitos se encontrem empregados, quer na situação em que os sujeitos se encontrem desempregados. Desta forma, a suposição inicial foi confirmada, na medida em que a frequência e conclusão de cursos de formação profissional diferencia os trabalhadores do mercado de trabalho instável em relação à sua perceção de saúde mental. Sustentados nos nossos resultados, parece-nos que a formação profissional promove a saúde mental, e isso parece ocorrer por via da geração do maior nível de expetativas de empregabilidade dos indivíduos que aderiram à formação - efeito motivação. Por quanto tempo essas expetativas positivas irão perdurar é algo que fica fora do alcance do nosso estudo. O gradiente de desmotivação dependerá das contingências futuras dos contextos de vida individuais. O que é sabido é que a financiarização da economia, que faz crescer de forma desregulada o "quarto mundo" de que fala Castells (1999), não permite estabelecer laços de continuidade entre a formação profissional e o emprego. E, por isso, é de prever que a diferença dos índices de saúde mental verificada entre os inquiridos seja, mais cedo ou mais tarde, anulada ou pelo menos alterada em função da situação em que cada um deles se venha a encontrar no mercado de trabalho. Reconhecemos, portanto, que a nossa pesquisa reclama a complementaridade de um estudo longitudinal. Em todo o caso, outros estudos, recorrendo ao mesmo instrumento para avaliar a saúde mental de trabalhadores em situação de desemprego, mostraram indícios que relacionam essa situação com a saúde precária do trabalhador desempregado (BARROS; OLIVEIRA, 2009) ou mesmo como causa de deterioração do bem-estar psicológico (ARGOLO; ARAÚJO, 2004), apontando "para a necessidade de estudos mais abrangentes que possam subsidiar políticas públicas e estratégias de enfrentamento do problema circunscrito à relação entre homem e trabalho" (BARROS; OLIVEIRA, 2009, p. 98). Esta necessidade é, de resto, partilhada por outros autores (e.g. BERNARDO et al., 2011), quando se reportam à formulação de políticas públicas na área da saúde do trabalhador.

Seja como for, a ideia de que a formação profissional poderá ser uma boa estratégia de coping para os indivíduos em idade ativa ganha força (MARTINS, 2004), pelo menos quando se reporta a um período temporal inferior a um ano. Do mesmo modo, a formação profissional, enquanto atividade educativa, estimula o relacionamento interpessoal, sendo esta uma condição básica para a boa saúde mental - efeito relacional. Se, por um lado, a saúde ocupacional se traduz numa maior motivação para o trabalho, satisfação profissional e produtividade, as estratégias de motivação dos trabalhadores, os contextos de satisfação e o envolvimento e participação produzem, igualmente, benefícios em termos de saúde ocupacional (SANTOS, 1996).

Para terminar, interessa deixar patente que esta investigação não pretende limitar-se à constatação empírica de uma diferenciação circunstancial. Intentamos assim fazer a contextualização da atual necessidade da formação profissional e, sobretudo, da atual relevância do sentimento de segurança que ela gera nos indivíduos. Dessa forma, inscrevemos o nosso contributo científico no imperativo ético da humanização do mercado de trabalho e consequente dignificação da condição dos trabalhadores (empregados e desempregados), os quais têm vindo a ser constantemente submetidos aos efeitos psicológicos negativos, resultantes da metamorfose laboral. Esta preocupação encontra paralelo noutras investigações, sendo de destacar a desenvolvida por Burke e Greenglass (2001, p.143), os quais enalteceram a importância da investigação e da ciência psicológica no que concerne a "fazer reestruturações de modo mais humano, desenvolvendo processos que concedam dignidade aqueles que irão perder o seu trabalho e reabilitação àqueles que permanecem". Martínez (1998, p. 209-210) acrescenta que para combater o stress laboral deve-se começar a pensar na Educação para a Saúde, concretamente: "a Educação para a Saúde se configura disciplinarmente como o âmbito do estudo e formação capaz de gerar processos de incidência social referentes à capacidade das populações para melhorar a qualidade da sua vida na sua dimensão mais ampla". Portanto, a presente investigação apenas procurou dar continuidade a um conjunto de esforços científicos já existentes, no que toca à criação de condições para que o mercado de trabalho não seja um elemento de stress para o trabalhador. Não podemos deixar de assinalar, em todo o caso, as limitações em termos de generalização de resultados, pelo recurso a uma amostra por conveniência. Propósitos inferenciais devem ser acautelados e nunca equacionados para populações com características económico-demográficas e socioculturais distintas.

Em trabalhos futuros, sugere-se o controlo do tempo de duração do(s) curso(s) de formação profissional em estudo e da sua especificidade no que diz respeito à área de formação em análise, incluindo o setor de emprego e o tipo de formação. A replicação do estudo noutros contextos, com o eventual recurso a análises multivariadas torna-se também desejável. Por último, a necessidade, de resto, já apontada, de outros estudos longitudinais, propiciadores da comparação da auto-perceção de saúde mental dos mesmos trabalhadores ao longo do seu ciclo laboral, permitirá uma compreensão mais aprofundada os efeitos da formação profissional, numa lógica de Aprendizagem ao Longo da Vida. 


\section{Contribuições de autoria}

Todos os autores tiveram contribuição substancial no projeto, no delineamento, no levantamento de dados ou na sua análise e interpretação; na elaboração do manuscrito ou contribuição importante na sua revisão crítica; e na aprovação final da versão publicada.

\section{Referências}

ÁLVARO, J. L. Desempleo y bienestar psicológico. Madrid: Siglo XXI, 1992.

ÁLVARO, J. L.; GARRIDO, A. Reflexos da situação laboral na saúde mental dos jovens. In: MENDES, A. M.; BORGES, L. O.; FERREIRA, M. C. (Org.). Trabalho em transição, saúde em risco. Brasília: UnB, 2002. p. 207-224.

ÁLVARO, J. L.; TORREGROSA, J. R.; LUQUE, A. G. Estructura social e saúde mental. In: __ (Org.). Influencias sociales e psicológicas en la salud mental, Madrid: Siglo XXI, 1992, p. 9-30.

ARGOLO, J.C.; ARAÚJO, M.A. O Impacto do Desemprego sobre o Bem-Estar Psicológico dos Trabalhadores da Cidade de Natal. Revista de Administração Contemporânea, Rio de Janeiro, v. 8, n. 4, p. 161-182, out./dez. 2004.

BANKS, M. H. Desempleo y salud mental: investigaciones británicas recientes. In: ÁLVARO, J. L.; TORREGROSA, J. R.; LUQUE, A. G. (Org.). Influencias sociales y psicológicas en la Salud Mental, Madrid, Siglo XXI, 1992, p. 143-170.

BARROS, C.; OLIVEIRA, T. Saúde mental de trabalhadores desempregados. Revista Psicologia: Organizações e Trabalho, Brasília, v. 9, n. 1, p. 86-107, jan./jun. 2009.

BERNARDO, M. H et al. Ainda sobre a saúde mental do trabalhador. Revista Brasileira de Saúde Ocupacional, São Paulo, vol. 36, nº 123, p. 8-11, jan./jun. 2011.

BLANCH, J. M. Del viejo al nuevo paro. Un análisis psicológico e social. Barcelona: Promociones y Publicaciones Universitarias, 1990.

BUENDÍA, J. Desempleo y salud mental. In: BUENDÍA, J.; RAMOS, F. (Org.). Empleo, estrés y salud. Madrid: Edições Pirâmide, 2001. p. 147-160.

BURKE, R. J.; GREENGLASS, E. R. Reestructuración organizacional, reducción de plantilla y bienestar laboral. In: BUENDÍA, J.; RAMOS, F. (Org.). Empleo estrés y salud. Madrid: Edições Pirâmide, 2001. p. 131-146.

CASTELLS, M. La era de la información, vol. III, Fin de milenio, Madrid: Alianza Editorial, 1999.

FERNANDÉZ, F. Modelos actuais de educação de pessoas adultas. In: CANÁRIO, R.; CABRITO, B. (Org.). Educação e formação de adultos: mutações e convergências. Lisboa: Educa, 2005.
FERREIRA, M. C. Marcas do Trabalho e Bem-estar no Serviço de Atendimento ao Público. In: MENDES, A. M.; BORGES, L. O.; FERREIRA, M. C. (Org.). Trabalho em transição, saúde em risco. Brasília: UnB, 2002.

GAZIER, B.: Assurance-chômage, employabilité et marches transitionnels du travail. Paris : Université Paris 1, 1999.

GLINA, D. M. R. et al. Saúde mental e trabalho: uma reflexão sobre o nexo com o trabalho e o diagnóstico, com base na prática. Cadernos de Saúde Pública, Rio de Janeiro, v. 17, n. 3, p. 607-616, maio/jun. 2001.

GOLDBERG, D. P.; HILLIER, V. F. A scaled version of the General Health Questionnaire. Psychological Medicine, v. 9, p. 139-145, february, 1979.

HARNOIS, G.; GABRIEL, P. Mental health and work: Impact, issues and good practices. Geneva: WHO Press, 2000.

MARÔCO, J. Análise Estatística - com o PASW Statistics (Ex-SPSS). Pêro Pinheiro: ReportNumber, 2010.

MARÔCO, J.; BISPO, R. Estatística aplicada às ciências sociais e humanas. Lisboa: Climepsi, 2005.

MARTÍNEZ, A. G. Estrés laboral y educación para la salud. In BUENDÍA, J. (Org.). Estrés laboral y salud. Madrid: Editorial Biblioteca Nueva, 1998. p. 207-220.

MARTINS, M. C. A. Factores de risco psicossociais para a saúde mental. Millenium, n. 29, p. 255-268, 2004.

MONTEIRO, A. P. Assessment of the factor structure and reliability of the Portuguese version of the General Health Questionnaire-28 among adults. Journal of Mental Health, v. 20, no 1, p. 15-20, 2011.

ORGANIZAÇÃO MUNDIAL DE SAÚDE (2001). Relatório sobre a Saúde no Mundo. Saúde Mental: Nova Concepção, Nova Esperança. Disponível em: $<$ http://www.dgsaude.pt/upload/membro.id/ficheiros/ i006020.pdf > . Acesso em: 8 de Novembro de 2004.

RIBEIRO, J. L. P. Introdução à Psicologia da Saúde, Coimbra: Quarteto, 2005.

RIBEIRO, J. L. P.; ANTUNES, S. Contribuição para o estudo de adaptação do Questionário de Saúde Geral de 28 Itens (General Health Questionnaire-GHQ28).

Revista Portuguesa de Psicossomática, v. 5, n. 1, p. 3745, 2003.

RIPOLL, P.; RODRIGUEZ, I.; PEIRÓ, J. M. Desempleo. In: PEIRÓ, J. M.; PRIETO, F. (Org.) Tratado de psicología del trabajo: aspectos psicosociales del 
trabajo. Volumen. II. Madrid: Editorial Síntesis, 1996. p. 225-247.

SANTOS, E. Saúde ocupacional. Interacções, 3, p. 6974, 1996.

STIGLITZ, J. E. Globalização: a grande desilusão. Lisboa: Terramar, 2004.
UNIÃO EUROPEIA. Decisão no 2493/95/CE, de 23 de Outubro de 1995. Proclama 1996 «Ano Europeu da Educação e da Formação ao Longo da Vida». Parlamento Europeu e do Conselho, 23 de out. 1995. Disponível em: <http://eur-lex.europa.eu/legalcontent/PT/TXT/PDF/?uri=CELEX:31995D2493\&from $=\mathrm{PT}>$. Acesso em: 01 jul. 2014. 